\title{
Acute Cortical Necrosis
}

National Cancer Institute

\section{Source}

National Cancer Institute. Acute Cortical Necrosis. NCI Thesaurus. Code C123163.

Acute kidney injury caused by ischemic necrosis of the renal cortex. 